\title{
Part of the fabric and mostly right: an ethnography of ethics in clinical practice
}

\author{
Although \\ ethical \\ problems were \\ only rarely \\ discussed \\ specifically, \\ they were \\ generally \\ regarded as an \\ integral aspect \\ of patient care
}

Evan Doran $B A, P h D^{1.2}$

Jennifer Fleming

BA, MHA, PhD

Christopher Jordens BA (Hons), MPH, PhD

Cameron L Stewart $\mathrm{BEc}, \mathrm{LLB}(\mathrm{Hons}), \mathrm{PhD}^{3}$

Julie Letts

MBioeth $^{4}$

Ian H Kerridge MPhil, FRACP, FRCPA

IUniversity of Newcastle,

Newcastle, NSW.

2 Centre for Values, Ethics

and the Law in Medicine,

University of Sydney,

Sydney, NSW.

3 University of Sydney,

Sydney, NSW

4 NSW Ministry of Health,

Sydney, NSW.

evan.doran@

newcastle.edu.au

doi: 10.5694/mjal4.00208

Perspective p 568

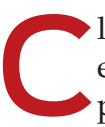

linical ethics support is an emerging field of theory and practice concerned with enhancing the ethical quality or "ethicality" of clinical practice (rather than research) in hospitals and other health care institutions. ${ }^{1,2}$ Clinical ethics support is typically delivered by a multidisciplinary ethics committee, an individual ethicist, or both. Its aim is to provide informed advice for the development of organisational policies, as part of staff education, and in ethically difficult situations that arise in the institution.

Clinical ethics support services are an established feature of health care in the United States and Canada, and are becoming more common in the United Kingdom and elsewhere in Europe and Asia. Although such programs have not been systematically evaluated, observational and experimental studies indicate that clinicians find them helpful, and that they reduce conflict, save money and improve the overall quality of patient care. ${ }^{3-8}$ While there has been grassroots enthusiasm for the development of clinical ethics support services in Australia (they are already available in some hospitals ${ }^{9-12}$ ), they have not yet been widely introduced and generally receive little, if any, institutional support.

There is limited evidence on which to base decisions about clinical ethics support, as there have been few empirical investigations of ethics in health care settings in Australia. It is therefore difficult to know whether clinical ethics support services are either wanted or necessary, or what would be required for such services to be established and flourish. Our aim in this study, part of a research project funded by the New South Wales Government, was to describe ethics as it is practised in one health care setting, and to ascertain whether health care professionals support the establishment of clinical ethics support services.

\section{Abstract}

Objectives: To describe how ethics is practised in a health care setting, and to ascertain whether there was interest in establishing clinical ethics support services.

Design and setting: Observations and interviews undertaken between April and November 2012 in a large NSW urban hospital with newborn care, maternity and oncology departments and analysed by coding and categorising the data.

Main outcome measures: Key themes in the participants' attitudes to professional ethics were identified.

Results: Ethics is not typically an explicit feature of clinical deliberations, and clinicians tend to apply basic ethical principles when ethical problems are identified. They also discuss difficult decisions with colleagues, and try to resolve ethical differences by discussion. Participants judged the ethics of clinical practice to be "mostly right", primarily because ethics is "part of the fabric" of everyday clinical work that aspires to "optimising care". Nevertheless, most clinicians would welcome ethics support because ethics is integral to health care practice, is not always "done well", and may be the source of conflict.

Conclusions: Ethics is very much a part of the fabric of clinical practice, and the ethical challenges that arise in patient care in this particular setting are generally managed adequately. However, many clinicians have concerns about the ethical aspects of some practices and decisions, and believe that access to expert ethics support would be useful. Helping clinicians to provide ethically sound patient care should be a priority for health care providers across Australia.

\section{Methods}

We wanted to qualitatively describe the "ethical environment" of a clinical setting; that is, the ethical difficulties that arise and how they are managed. We therefore took an ethnographic ("first-hand empirical investigations of social organisation and culture ${ }^{\prime \prime 13}$ ) approach: we observed clinicians and talked to them about ethical problems in everyday clinical practice, about their satisfaction with current processes for dealing with ethical questions, and about whether they felt that clinical ethics support services would be useful.

The study was conducted between April and November 2012 in a large NSW urban hospital with newborn care, maternity and oncology departments. Data collection involved one researcher visiting wards, observing clinical meetings (including handovers, case review meetings, and morbidity and mortality meetings), and conducting impromptu and formal interviews with key participants. We selected men and women from a variety of professions, seniority levels, age groups and ethnic backgrounds. Observations and impromptu interviews were recorded in field journals, and formal interviews were electronically recorded for later transcription. The formal interviews were guided by a set of broad questions, such as: "What aspects of patient care involve ethical questions? Is it easy to discuss an ethical problem when you want or need to?" Follow-up questions probed for detail or clarification.

Analysis involved coding and categorising the data, and identifying key themes in the participants' attitudes to professional ethics. Initial analysis was undertaken by two researchers who independently reviewed the data and then compared and clarified interpretations. Analysis focused on what clinicians said about ethics management and how they thought it could be improved. Areas of convergence and divergence of opinion 
Selected quotes from interviewed health professionals that illustrate the major themes that emerged in their descriptions of clinical ethics in their hospital or unit

\section{Mostly right}

“.... we do get it right. Every so often you won't, but I think that's a normal process and I think that you can't guarantee that you get it right $100 \%$ of the time. But I think the multidisciplinary input into those sort of decisions make it as right as it can be." Interviewee 24

\section{Optimising}

"Well, what's important to me is that women come out of here with as healthy a baby as they can have and without something bad happening to them that I could have prevented." Interviewee 8

"It's important for people to be accountable, right? I'm accountable to the people who work with me, to those people who I supervise ... So, if I make a decision, I need to be able to give them a good reason for that." Interviewee 3

"For me here ethically, it's to do the right and the best thing for the women ... that we're honest and we're open and we support them regardless of what it is that I might bring to work, that you're supporting choice." Interviewee 1

"... we are not there to judge, we are there to do the job." Interviewee 29

\section{Part of the fabric}

"So I guess it's about whether or not it's woven into your culture. But you're right, are people sitting there and saying well, are we balancing the ethics of this particular situation or not? So they're not actually bringing it up as such and labelling it. But it's certainly there." Interviewee 25

"A lot of times we are practising, and we don't verbalise it ... there are other things ... to consider all the factors, all the other things and yes, it's all about the ethics and all those things but we don't verbalise ... we are practising it." Interviewee 30

"... that is a hard call and the way you actually go and take the decision is not an individual decision, so you involve all your other colleagues as well as the nursing colleagues, senior colleagues when you make those particular decisions." Interviewee 17

"[Team work is] very important, because what it means is that we're all walking together down the same path ... whether it's in terms of managing a baby or ... whether it's doing anything, any clinical work itself ... we do try to be democratic as much as we can be. In a big unit like that, sometimes you cannot. But we do strive towards that." Interviewee 29

\section{Moral disquiet}

"Sometimes I'm just left sitting there in a meeting going 'Oh my God, I can't believe that,' but I don't always feel very comfortable questioning a medical decision unless it really is in my court." Interviewee 15

"It gets difficult, clearly, however, over the issue of terminations and because they're increasing in number ... we are finding more and more things, which we think are not the best for the baby ... we had a case last year where a woman ended up having a foeticide at 26 weeks. Now, the circumstances surrounding that for me were absolute anathema ... There was to my great disappointment a matter of factness about it. To the point where it was difficult for me to even say much more. But that's the current climate." Interviewee 3

\section{Clinical ethics support}

"I think it would be really valuable for us to have a formal ethics committee. That would be really, really valuable for us ... to have all the lead-up preliminary discussions where people are prompted into thinking more deeply." Interviewee 25

"I don't think there's been anything where l've thought that we could've needed some ethical support. I think that we are pretty good on that." Interviewee 14

“... it's not as if they can say that you do or don't, that you should or shouldn't do something but it's hard to know what role can they actually have and how can an ethics committee help." Interviewee 23

were identified, and themes that represented the participants' assessment of ethics in everyday clinical practice were distilled as phrases, such as "mostly right". Regular meetings of the wider research group reviewed the analysis and discussed emerging themes.

The study was approved by the Hunter New England Human Research Ethics Committee (10/12/15/4.12) and the NSW Human Research Ethics Committee (HREC/10/HNE/373).

\section{Results}

A total of 30 semistructured interviews with 11 medical staff, 18 nursing and midwifery staff, and one psychologist were conducted; 24 of the interviewees were women, 6 were men. In clinical meetings, staff discussed their patients; they updated, reviewed and justified plans for their care. In conversations and interviews, clinicians described difficult cases and problems, and offered explanations about how these were generally managed. In the following discussion we present the main themes of our analysis; the Box includes a selection of representative raw statements by the participants.

\section{Mostly right}

Clinicians' accounts conveyed the impression that they assessed ethics management as being "mostly right", and that they were generally satisfied with the ethics of the hospital, their division and their colleagues. Although ethically difficult situations and decisions often arose, they did not usually cause personal distress or lead to disputes or disruption.

In most accounts, clinicians described themselves and their colleagues as honest, compassionate, nonjudgemental and respectful of their patients' agency and autonomy. They believed that the ethics of clinical care was generally handled well, primarily because ethics was perceived as "woven into" the culture of the hospital, and also because clinicians assumed that their colleagues were competent in dealing with basic moral questions and aimed to optimise patient care.

\section{Optimising}

The continuous and collective process of deciding what was best for individual patients and acting accordingly dominated clinicians' accounts of their activities, and this was supported by our observations. The shared primary imperative was to optimise patient care; that is, to balance the benefits and risks of different options, while considering the individual patient's preferences and taking into account the available resources.

Optimisation was also clearly evident in clinicians' descriptions of the routines, procedures, guidelines and policies that guided practice. In the pursuit of these objectives, clinicians and clinical teams and units tended to reflect in both word and practice fundamental bioethical principles (beneficence, non-maleficence, autonomy and justice) and concepts (vulnerability, equity, safety), although these were not always explicitly mentioned or discussed. 
While mistakes and lapses in judgement were described by participants, most accounts emphasised that both individual clinicians and the clinical team were doing their professional best; that is, consistently applying their clinical knowledge and skills, and recognising that they were accountable for the outcomes of their decisions and actions. Weighing up what was best for patients included considering the available evidence on best practice, clinical experience, and the patients' preferences.

\section{Part of the fabric}

Clinical ethics was a pervasive but often unspoken part of everyday clinical work. Clinicians were always "doing" ethics, but only infrequently in an explicit manner. Although ethical problems were only rarely discussed specifically, they were generally regarded as an integral aspect of patient care. When decisions were needed or questions of moral significance arose, they were generally handled according to established policies and procedures, or by seeking the opinions of colleagues.

For difficult cases or "hard calls", participants attach great importance to allowing all members of the team to contribute to decisions about patient care. While an open democratic process was not always possible and consensus not always achieved, drawing on "collective wisdom" (as expressed in established policies and procedures, the opinions of colleagues and team discussions) was perceived by most participants as a sound basis for ensuring that the ethics of patient care was "mostly right".

\section{Moral disquiet}

Although ethical disputes were infrequent, clinicians often reported having ethical concerns and experiencing moral disquiet or occasionally even distress about a particular case or an aspect of patient care. The kinds of situations described as ethically troubling included late (third trimester) terminations of pregnancy, "futile" or "aggressive" treatments, interventions during childbirth, patients refusing recommended treatments and patient confidentiality.
While some clinicians said that they were able to voice their concerns to others, junior clinicians and non-medical health practitioners, in particular, reported that it was not always possible or practical to speak out about a problem or decision that concerned them, or on one with which they disagreed on ethical grounds. A number of conditions appeared to constrain acting on or airing one's moral disquiet, including proximity to the decision, the role and authority of the individual, uncertainty surrounding the material facts of the case (eg, the likelihood of good or bad outcomes), and the reluctance (particularly of nurses and junior medical staff) to challenge a senior colleague, to "rock the boat" or to create disharmony in the team because one disagreed with the prevailing consensus.

\section{Clinical ethics support}

Although conditions were "mostly right", ethical concerns that arise in health care situations were sufficiently substantial that most of those with whom we spoke favoured the idea of clinical ethics support, even though, as had been expected, most had neither heard of nor had any experience with such services. Some participants, however, could not imagine how clinical ethics support would assist them, or were sceptical about its potential utility.

\section{Discussion}

Our observations concur with those of other qualitative studies of clinicians and everyday clinical ethics. Our data, like that of a previous study, ${ }^{14}$ indicate that all clinical work carries some ethical "charge", and that "principles" describe how clinicians approach and explain the ethics of patient care. It was clear that this "charge" only occasionally results in conflict, and ethical differences between clinicians, or between clinicians and patients and their families, were resolved by discussion, as also previously found. ${ }^{15}$

The clinicians in this study also reported managing more difficult ethical situations in ways that have been observed elsewhere, primarily by seeking the opinions and assurances of their colleagues. ${ }^{16}$ For the most part, however, as also reported by other authors, ethics was not typically an explicit feature of clinical practice or of clinical deliberations. ${ }^{15-18}$ While overt conflict was infrequent, many clinicians experienced moral disquiet or uncertainty about particular decisions or practices.

Most of the participants in this study judged the ethics of clinical practice to be "mostly right" because it was "part of the fabric" of everyday clinical work that aspired to "optimise" patient care. This may be a reflection of the "settled morality" that can emerge in a particular clinical setting. "Settled morality" refers to the general agreement within a group about what is right and wrong when dealing with frequently encountered ethical questions. ${ }^{15}$ The settled morality in a health care institution is articulated to a large extent in the multitude of hospital policies and guidelines that regulate the interactions of clinicians, and those of clinicians and patients. Ethical values or principles can be explicitly included in such guidelines and policies; more frequently, however, ethical values, concepts and principles, such as care, respect, safety, quality, veracity, transparency, vulnerability, justice and equity are implicitly embedded in an institution's policies and guidelines, providing clinicians with a framework for responding to ethically difficult cases. ${ }^{19}$

The assessment that ethics in clinical care is "mostly right" recognises that it sometimes falls short of being "right". It is naturally dangerous to be too tolerant of imperfection by, for example, overlooking rather than tackling difficult but important ethical problems. The relative infrequency of moral conflict could reflect the fact that, within the settled morality, clinicians become inured to many of the ethical aspects of patient care. Others have argued that the problem of settled morality in health care is that ethics can become reduced to routine and normalised to the point of invisibility. ${ }^{15}$ Indeed, a settled morality does not guarantee inclusive ethical decision making, even if meetings are relatively open. As 
we observed, even profound moral disquiet is not always expressed. This can be a source of frustration for clinicians and may contribute to "moral distress"; that is, the potential or actual threat to the moral agency and integrity of an individual clinician, ${ }^{20,21}$ which, if not appropriately managed, can have a negative effect on staff morale and the quality of care. $^{22-25}$

We undertook this study in a single specialised hospital, and our findings may not apply to other settings. Ethical challenges, and how they are and can be managed, will vary between specialist clinical areas, with specific problems arising in areas such as mental health, geriatrics and emergency medicine. Further qualitative investigation of other settings and specialties is needed to expand and develop these initial findings and to gain further insights into everyday clinical ethics and preferences about clinical ethics support.
Further, as our study canvassed only the views of the medical staff, we do not know whether patients and their families would evaluate the ethics of care in this hospital as "mostly right". The process of establishing and monitoring the operation of clinical ethics support must also involve their perspective. Further qualitative research involving patients and their families should be undertaken. Routine patient satisfaction and discharge surveys could include questions related to the ethics of patient care.

Although our study involved only a single setting, there is no reason to believe that the situation in other Australian hospitals would be greatly different. That clinicians are occasionally troubled by the ethical aspects of particular decisions or problems is unlikely to be surprising for most medical practitioners, regardless of their setting or clinical discipline. Similarly, our finding that the ethical aspects of a case or problem may not be recognised, openly discussed or satisfactorily managed is likely to resonate across a range of health care settings.

Our findings therefore suggest that helping clinicians to provide ethically sound patient care should be a priority for Australian public hospitals. We believe that clinical ethics support, expressly intended to raise awareness, encourage open discussion and minimise uncertainty and distress, should be considered as a means for providing this support. Finally, we also believe that serious consideration of clinical ethics support is ultimately a normative question, and should involve broad professional and public discussion on whether explicit engagement with ethics is integral to patient care in a democratic and inclusive society.

Acknowledgements: The study was funded by the NSW Ministry of Health. We acknowledge the support of all members of the Clinical Ethics Capacity Building Project Reference Group.

Competing interests: No relevant disclosures.

Received 13 Feb 2014, accepted 5 Feb 2015.

References are available online at www.mja.com.au. 
1 McLean SAM. Clinical ethics consultation in the United Kingdom. Diametros 2009; (22): 76-89.

2 Fox E, Bottrell MM, Berkowitz KA, et al. IntegratedEthics: An innovative program to improve ethics quality in health care. Innovation Journal 2010; 15(2): article 8.

3 La Puma J, Stocking CB, Silverstein MD, et al. An ethics consultation service in a teaching hospital: Utilization and evaluation. JAMA 1988; 260: 808-811.

4 La Puma J, Stocking CB, Darling CM, Siegler M. Community hospital ethics consultation: Evaluation and comparison with a university hospital service. Am J Med 1992; 92: 346-351.

5 McClung JA, Kamer RS, DeLuca M, Barber HJ. Evaluation of a medical ethics consultation service: opinions of patients and health care providers. Am J Med 1996; 100: 456-460.

6 Yen B, Schneiderman LJ. Impact of pediatric ethics consultations on patients, families, social workers, and physicians. J Perinatol 1999; 19: 373-378.

7 Schneiderman I, Gilmer T, Teetzel HD. Impact of ethics consultations in the intensive care setting: a randomized, controlled trial. Crit Care Med 2000; 28 : 3920-3924.

8 Schneiderman LJ. Effect of ethics consultations on nonbeneficial lifesustaining treatments in the intensive care setting: a randomized, controlled trial. JAMA 2003; 290: 1166-1172.

9 McNeill PM. A critical analysis of Australian clinical ethics committees and the functions they serve. Bioethics 2001; 15: 443-460.

10 Gill AW, Saul, P, McPhee J, Kerridge I. Acute clinical ethics consultation: the practicalities. Med J Aust 2004; 181: 204-206.

1 Gold H, Hall G, Gillam L. Role and function of a paediatric clinical ethics service: Experiences at the Royal Children's Hospital, Melbourne. J Paediatr Child Health 2011; 47: 632-636.

12 Delany C, Hall G. 'I just love these sessions'. Should physician satisfaction matter in clinical ethics consultations? Clin Ethics 2012; 7: 116-121.

13 Hammersley M, Atkinson, $P$. Ethnography: principles in practice (3rd edition). London: Routledge, 2007.

14 Robertson DW. Ethical theory, ethnography, and differences between doctors and nurses in approaches to patient care. J Med Ethics 1996; 22: 292-299.

15 Frith L, Jacoby A, Gabbay M. Ethical boundary-work in the infertility clinic. Sociol Health Illn 2011; 33: 570-585.

16 Hurst SA, Hull SC, DuVal G, Danis, M. How physicians face ethical difficulties: a qualitative analysis. J Med Ethics 2005; 31: 7-14.
17 Melia KM. Ethical issues and the importance of consensus for the intensive care team. Soc Sci Med 2001; 53: 707-719.

18 McGrath P, Henderson D. 2008, "Oh, that's a really hard question": Australian findings on ethical reflection in an accident and emergency ward. HEC Forum 2008; 20: 357-373.

19 Agich GJ. The issue of expertise in clinical ethics. Diametros 2009; (22): 3-20.

20 Epstein EG, Hamric AB. Moral distress, moral residue, and the crescendo effect. J Clin Ethics 2009; 20: 330-342.

21 Hamric AB. Empirical research on moral distress: issues, challenges, and opportunities. HEC Forum. 2012; 24: 39-49.

22 Schwenzer KJ, Wang, L. Assessing moral distress in respiratory care practitioners. Crit Care Med 2006; 34: 2967-2973.

23 Chiu PP, Hilliard RI, Azzie G, Fecteau A. Experience of moral distress among pediatric surgery trainees. J Pediatr Surg 2008; 43: 986-993.

24 Pendry PS. Moral distress: recognizing it to retain nurses. Nurs Econ 2007; 25: 217-221.

25 Pauly BM, Varcoe C, Storch J. Framing the issues: moral distress in health care. HEC Forum 2012; 24: 1-11. 